\title{
Molecular transitions as probes of the physical conditions of extragalactic environments ${ }^{\star}$
}

\begin{abstract}
Serena Viti
Department of Physics and Astronomy, University College London, Gower street, London, WC1E 6BT, UK

e-mail: serena.viti@ucl.ac.uk

Received 9 May 2016 / Accepted 29 August 2017

ABSTRACT

Aims. We present a method to interpret molecular observations and molecular line ratios in nearby extragalactic regions.

Methods. Ab initio grids of time dependent chemical models, varying in gas density, temperature, cosmic ray ionization rate, and radiation field, are used as inputs into RADEX calculations. Tables of abundances, column densities, theoretical line intensities, and line ratios for some of the most used dense gas tracers are provided. The degree of correlation as well as degeneracy inherent in molecular ratios is discussed. Comparisons of the theoretical intensities with example observations are also provided.

Results. We find that, within the parameters space explored, chemical abundances can be constrained by a well-defined set of gas density, gas temperature, and cosmic ray ionization rates for the species we investigate here. However, line intensities, and more importantly line ratios, from different chemical models can be very similar, thereby leading to a clear degeneracy. We also find that the gas subjected to a galactic cosmic ray ionization rate will not necessarily have reached steady state in 1 million years. The species most affected by time dependency effects are HCN and CS, which are both high density tracers. We use our ab initio method to fit an example set of data from two galaxies, i.e. M 82 and NGC 253. We find that (i) molecular line ratios can be easily matched even with erroneous individual line intensities; (ii) no set of species can be matched by a one-component interstellar medium (ISM); and (iii) a species may be a good tracer of an energetic process but only under specific density and temperature conditions.

Conclusions. We provide tables of chemical abundances and line intensities ratios for some of the most commonly observed extragalactic tracers of dense gas for a grid of models. We show that by taking the chemistry behind each species and the individual line intensities into consideration, many degeneracies that arise by just using molecular line ratios can be avoided. Finally we show that using a species or a ratio as a tracer of an individual energetic process, such as cosmic rays and UV, ought to be done with caution.
\end{abstract}

Key words. galaxies: active - astrochemistry - molecular processes - radiative transfer

\section{Introduction}

Following the first detection of a molecular species in external galaxies in the 1970s, molecular line emission studies have been routinely performed to determine the physical characteristics of nearby galaxies (e.g. Takano et al. 2002; Martín et al. 2009; Aladro et al. 2011, 2013). Galaxies span a variety of shapes, sizes, and physical conditions. The physical parameters, such as gas densities, ultraviolet (UV) fields, cosmic ray ionization rates, and dust properties, which determine the appearance of our own Galaxy may therefore be very different in other galaxies. Nevertheless external galaxies, in general, contain the variety of regions and sources similar to those that we can identify in the Milky Way; hence molecules could be ideal probes of the dense gas that is the reservoir for star formation and could help in describing the process of star formation itself as well as the influence of newly formed stars on their environments (Williams \& Viti 2014).

Because of their range of critical densities across the different molecular species and across the transitions of the same molecule, molecular observations are an ideal tool to trace a wide range of gas densities in the interstellar medium and the dependencies of chemical reactions on the energy available to the system, i.e. the kinetic temperature of the gas. However, what makes observations of molecules so adept is

\footnotetext{
* Tables 2-11 are only available at the CDS

via anonymous ftp to cdsarc.u-strasbg. fr (130.79.128.5) or via http://cdsarc.u-strasbg.fr/viz-bin/qcat?]/A+A/607/A118
}

also what complicates their interpretation. In particular, even with the highest spatial resolution available with the Atacama Large Millimeter/Submillimeter Array (ALMA), the beam usually encompasses emissions from many types of regions and the molecular emission is an ensemble of multi-phase gas in which the spatial and temporal effects are diluted in the beam. Concomitant detection of multiple molecules in a spatially unresolved galaxy therefore does not necessarily mean that they are emitted from the same gas (e.g. Viti 2015). While this is not surprising, many multi-species studies of nearby galaxies still need to assume coexistence of molecular emission when interpreting their observations (e.g. Usero et al. 2004; Krips et al. 2011; Viti et al. 2014). This is partly because we are forced to use multiple transitions and molecules to overcome the wellknown degeneracy between density and temperature for linear molecules, which implies that single transitions of these molecules cannot be easily used to determine quantitatively the gas density or temperature. There have been several theoretical studies on the sensitivity of chemistry to the range of physical parameters that determine a galaxy (e.g. Bayet et al. 2008, 2009, 2011; Meijerink et al. 2011; Kazandjian et al. 2012, 2015). Most of these sensitivity studies concentrated on the modelling of photon-dominated regions (PDR) or X-ray dominated regions (XDR), rather than the modelling of dense molecular gas. Observationally, many studies have shown that molecular line ratios, such as $\mathrm{HCO}^{+} / \mathrm{HCN}$ or $\mathrm{HCN} / \mathrm{CO}$, differ across different types of galaxies especially between active galactic nuclei (AGN)-dominated galaxies and starburst-dominated galaxies 
A\&A 607, A118 (2017)

Table 1. $\mathrm{CH}_{3} \mathrm{OH}$ and $\mathrm{HNCO}$ column densities at two different times for shock models only.

\begin{tabular}{|c|c|c|c|c|c|c|}
\hline \multirow[t]{2}{*}{$M$} & Time & $N\left(\mathrm{CH}_{3} \mathrm{OH}\right)$ & $N(\mathrm{HNCO})$ & \multirow{2}{*}{$\begin{array}{l}\text { Time } \\
\text { Years }\end{array}$} & $N\left(\mathrm{CH}_{3} \mathrm{O}\right.$ & \multirow{2}{*}{$N(\mathrm{HNCO})$} \\
\hline & Years & \multicolumn{2}{|c|}{$\mathrm{cm}^{-2}$} & & $\mathrm{~cm}^{-2}$ & \\
\hline 65 & 760 & $1.5(16)$ & $5.3(13)$ & $10^{5}$ & $7.6(14)$ & $5.6(13)$ \\
\hline 66 & 70 & $1.1(18)$ & $3.5(12)$ & $10^{5}$ & $2.5(16)$ & $3.0(13)$ \\
\hline 67 & 7 & $3.7(17)$ & $2.6(13)$ & $10^{5}$ & $1.5(16)$ & $1.1(14)$ \\
\hline
\end{tabular}

Notes. The first time corresponds to a time when the temperature of the gas reaches its maximum, which is different among models. $a(b)$ stands for $a \times 10^{b}$.

(e.g. Kohno et al. 2005; Krips et al. 2008), although some studies have found that enhanced $\mathrm{HCN} / \mathrm{HCO}^{+}$are not unique to $\mathrm{AGN}$ environments but can also be found in systems dominated by star formation (e.g. Privon et al. 2015). However the derived abundance ratios for an individual galaxy also highly differ across studies depending on the transitions observed, available resolution, and method used in deriving the column densities. An understanding of the chemistry behind each molecule and its dependencies on the density and temperature of the gas is therefore essential and will lead to a more coordinated approach to understanding the nature of galaxies using molecular line emissions.

In this paper we use, as a test bed, a relatively small grid of chemical models to present a method for the interpretation of molecular observations and molecular line ratios in nearby extragalactic regions, in particular, starburst and AGN dominated galaxies as these are the most targeted types of galaxies for dense gas studies; similar studies can be repeated for ultra luminous infrared galaxies (ULIRG), dwarf galaxies, etc., where, however, much of the gas may be in ionic or atomic form (e.g. van der Werf et al. 2010; Williams \& Viti 2014). We analyse the theoretical abundances and column densities of some of the most common tracers for a parameter space in gas density, temperature, cosmic ray ionization rate, and radiation field. The theoretical abundances are then used as inputs to the radiative transfer code RADEX (van der Tak et al. 2007), which in turn provides the theoretical intensities as well as common line ratios for the parameter space investigated by the chemical models. We investigate the degree of correlation and degeneracy inherent in each ratio and show how one can use theoretically derived abundances and line intensities for comparison with observations by using published observational data as examples.

The aim of this study is twofold. First, we seek to aid the observer in determining the most likely scenario that fits the data when multiple solutions are present. Second, we provide Tables 1-11, A.1-A.5 that one can use directly to compare with their observed intensities and intensity ratios for the most common used transitions; the tables are simply meant to be an example subset but include some of the most observed molecular species and transitions. We present the chemical models and discuss their degeneracies in Sect. 2. In Sect. 3 we first calculate theoretical line intensities and line ratios for four of the most observed gas tracers: $\mathrm{CO}, \mathrm{HCN}, \mathrm{HCO}^{+}$, and $\mathrm{CS}$. While the focus of this paper is purely on the characterization of the dense gas, $\mathrm{CO}$ (whose critical density is low and hence ubiquitously traces all gas above $\sim 100 \mathrm{~cm}^{-3}$ ) is included because it is still routinely used to interpret molecular ratios or determine the star formation rate density (e.g. Kohno et al. 2001, 2005; Tan et al. 2013), especially if high- $J$ CO observations are available (Zhao et al. 2016). Secondly, for completeness, we also extend our abundance and line intensities analyses to further species, not as commonly observed in external galaxies as those above, but often used as tracers of particular energetic events (e.g. shocks). In Sect. 4 we make use of our calculations to partly reinterpret observations in two very well studied galaxies to give examples of how this methodology can be used.

\section{Chemical models: Theoretical molecular abundances}

We used the UCL_CHEM ${ }^{1}$ gas-grain time dependent chemical code (Viti et al. 2004) to run a grid of chemical models covering a range of gas densities $\left(10^{4}-10^{6} \mathrm{~cm}^{-3}\right)$, temperatures $(50-$ $200 \mathrm{~K}$ ), radiation fields (1-500 Draine), cosmic ray ionization rates (1-500 standard galactic cosmic ray ionization field), and visual extinctions (1-50 mag); we used the cosmic ray ionization flux to simulate an enhancement in X-ray flux. As previously noted (Viti et al. 2014), this approximation has its limitations in that the X-ray flux heats the gas more efficiently than cosmic rays. However, the chemistry arising from these two fluxes should be similar.

While there are several more parameters that can be explored when performing chemical modelling, for the purpose of this study we chose those that affect most the line intensities of dense gas tracers in starburst and AGN-dominated galaxies, as predicted by large velocity gradient (LVG) models. For example, gas density, temperature, and visual extinction (directly correlated to column density) are all input parameters to RADEX and other LVG models. Cosmic ray ionization and radiation fluxes are known to differ from the Galactic values in starburst and AGN-dominated galaxies, and hence are also considered. Moreover they directly affect the temperature of the gas and dust. The choice of the lower and upper limits for the gas densities and temperatures reflect the best fits found for the dense molecular gas in the most commonly studied starburst and AGN galaxies; they are by no means meant to represent the only possible values. The range of cosmic ray ionization rates and radiation fields are subsets of the wider range explored in previous theoretical studies. As already mentioned (e.g. Bayet et al. 2009, 2011; Meijerink et al. 2013), our grid of models is not meant to be exhaustive but simply represent a plausible range of physical conditions. We also ran some models simulating the presence of shocks. For these models we adopted a representative shock velocity of $40 \mathrm{~km} \mathrm{~s}^{-1}$, which depending on the pre-shock densities, leads to a maximum temperature for the shock ranging from $2200 \mathrm{~K}$ to $4000 \mathrm{~K}$. The shocks in UCL_CHEM are treated in a parametric form as in Jimenez-Serra et al. (2008). Below we briefly describe UCL_CHEM. Further details of this version of UCL_CHEM, coupled with the shock module, can be found in Viti et al. (2011), and Holdship et al. (2017).

UCL_CHEM is an ab initio model and computes the evolution as a function of time of chemical abundances of the gas and on the ices starting from a diffuse and atomic gas (at a density of $10 \mathrm{~cm}^{-3}$ ). The parameter regulating the visual extinction

\footnotetext{
1 This code has now being made public (Holdship et al. 2017) and can
} be downloaded at https://uclchem.github.io/ 
has two effects on the models. First, this parameter affects the photochemistry since the radiation field is very efficiently attenuated for $A_{V}>4-5 \mathrm{mag}$. However, and more importantly, this paramater is effectively a measure of the size of the emission region, since in order to derive column densities from the fractional abundances calculated by the models we multiply the latter by the hydrogen column density as measured at 1 mag times the visual extinction. This is the so-called "on the spot" approximation (e.g. Dyson \& Williams 1997). As the chemical model is single point, we estimate the theoretical column densities, a posteriori.

The code is run in two phases in time (Phase I and Phase II). The initial density in Phase I is $\sim 10 \mathrm{~cm}^{-3}$. The gas is allowed to collapse and to reach a high density gas by means of a free-fall collapse. The collapse is not meant to represent the formation of protostars, but it is simply a way to compute the chemistry of high density gas in a self-consistent way starting from a diffuse atomic gas, i.e. without assuming the chemical composition at its final density. Of course, it is unknown how the gas reached its high density and we could have assumed any other number of density functions with time. For simplicity, we adopt free-fall collapse. The temperature during this phase is kept constant at $10 \mathrm{~K}$, and the cosmic ray ionization rates and radiation fields are at their standard Galactic values of $\zeta_{0}=5 \times 10^{-17} \mathrm{~s}^{-1}$ and 1 Draine, or $2.74 \times 10^{-3} \mathrm{erg} / \mathrm{s} / \mathrm{cm}^{2}$, respectively. In this way the chemistry of the high density gas is computed in a self-consistent way starting from a diffuse atomic gas, i.e. without assuming the chemical composition at its final density. During Phase I atoms and molecules are allowed to freeze onto the dust grains and react with each other, forming icy mantles. In Phase II, for the non-shock models, UCL_CHEM computes the chemical evolution of the gas and the icy mantles after either an assumed burst of star formation or AGN activity has occurred. The temperature of the gas increases from $10 \mathrm{~K}$ to a value set by the user and sublimation from the icy mantles occurs. The chemical evolution of the gas is then followed for $10^{7} \mathrm{yr}$. In the shock models, Phase II considers a plane-parallel C-Shock propagating with a velocity, $V_{\mathrm{s}}$, through the ambient medium. Details of the code and its use for the determination of abundances in external galaxies can be found in Viti et al. (2014). A detailed description of the shock module in UCL_CHEM can be found in Viti et al. (2011) and in Jimenez-Serra et al. (2008). In both phases of the UCL_CHEM model, the gas phase chemical network is based on the UMIST13 database (McElroy et al. 2013) with updates from the KIDA database (Wakelam et al. 2015). The surface reactions included in this model are assumed to be mainly hydrogenation reactions, allowing chemical saturation when possible.

Tables A.1 and A.2 list the grid of models with the resultant abundances (Table A.1), column densities, and ratios (Table A.2) for four of the most observed molecules in galaxies: $\mathrm{CO}, \mathrm{HCO}^{+}$, $\mathrm{HCN}$, and CS. Both tables show the results at the final time step $\left(10^{7} \mathrm{yr}\right)$. However for the shock models, the shock occurs at the beginning of Phase II and the models are stopped once the dissipation length has been reached; hence the Tables show the chemistry at the last time step, representing the post-shocked gas (at $\sim 10^{5} \mathrm{yr}$ ). For the shock models the $A_{V}$ is the initial visual extinction before the passage of the shock. In Table A.3 we tabulate the column densities for selected models at an earlier time of $1 \mathrm{Myr}$ (see Sect. 2.2).

Tables A.4, A.5, and Table 1 list the column densities for further species, often used as tracers of shocks (e.g. $\mathrm{SiO}, \mathrm{CH}_{3} \mathrm{OH}$, and SO) or surface grain chemistry (e.g. HNCO). HNC has been added because several studies use the $\mathrm{HNC} / \mathrm{HNC}$ ratio as a tracer of dense PDR and XDRs environments (e.g. Green et al. 2016). We note that $\mathrm{HNCO}$ and $\mathrm{CH}_{3} \mathrm{OH}$ do not survive until chemical equilibrium is reached and they are only listed for $10^{6} \mathrm{yr}$ for all non-shock models. Table 1 lists the column densities for $\mathrm{CH}_{3} \mathrm{OH}$ and $\mathrm{HNCO}$ for Models 65-67 at two different times: during the peak temperature of the shock and once the shock has passed. For the latter time the column densities were calculated assuming an average visual extinction of 10 mag.

\subsection{Chemical "degeneracy"}

From Tables A.1-A.5, and Table 1 we find a chemical degeneracy worth mentioning in relation to this study. Models differing by one or more parameters can still lead to column densities similar enough to be within the observational error in the column density estimation. For example, the HCN column density remains the same when cosmic ray ionization rate is enhanced from 1 to $10 \times$ the Galactic value (cf. Models 1 and 9); however this does not mean $\mathrm{HCN}$ is not sensitive to changes in the cosmic ray ionization rates as higher cosmic rate affect the HCN chemistry. In most cases this degeneracy can be dealt with if one observes multiple molecular species at the same spatial resolution. For example, Models 3 and 6 lead to almost identical CO, and similar $\mathrm{HCO}^{+}$and $\mathrm{HCN}$ column densities but they differ by $\sim$ one order of magnitude in CS. It is key to know a priori which species one needs to observe to differentiate among models by referring to Tables such as those presented here. However a more interesting degeneracy is caused when one obtains the same line intensities for different combinations of $\left[n_{\mathrm{H}}, T_{\text {gas }}, N(X)\right]$. We discuss this in Sect. 3.

\subsection{The importance of time dependency}

One chemical effect that is difficult to discern observationally is the time dependency of the abundance of each species. We do not discuss the shock models in this section, as chemical equilibrium is never reached during the passage of the shock. As shown by several studies in the past, not all chemistry is equally affected by time. Hence, while for some of the models we ran, equilibrium is reached well before 1 million years, for others it is not reached until much later; this is important because it is not obvious whether the large scale gas we observe, especially in starburst galaxies, will have remained subjected to the same energetic and dynamical conditions for longer than 1 million years because, for example, the flux of cosmic ray will vary across episodes of starbursts. Time dependencies effects have been discussed before (Bayet et al. 2008; Meijerink et al. 2013), in the context of specific galaxies as well (e.g. Aladro et al. 2013). In Tables A.3 and A.5 we tabulate the column densities for the models where chemical equilibrium has not been reached yet at 1 million years, and hence where the column densities differ from Tables A.2 and A.4. In Tables A.3 and A.5 there are no models with $\zeta$ higher than the standard value; it is clear therefore that a high cosmic ray ionization rate leads to steady state more quickly, as also found by previous work (Bayet et al. 2011). On the other hand, an enhanced radiation field (at least up to 500 the interstellar galactic field) does not help the chemistry in reaching steady state at the visual extinctions considered here, since the UV does not penetrate. Interestingly, of the four most observed species $\left(\mathrm{CO}, \mathrm{HCN}, \mathrm{HCO}^{+}\right.$, and $\left.\mathrm{CS}\right)$, the species most affected by time dependency is HCN followed by CS: both of these species are routinely used as high density gas tracers.

Some species are always short-lived; in the parameter space explored, these are $\mathrm{CH}_{3} \mathrm{OH}$ and $\mathrm{HNCO}$. In all the non-shock models these two species are always destroyed by the time chemical equilibrium is reached. In models not listed in Table A.5, 
these species do not even survive for a $1 \mathrm{Myr}$; hence an observed high abundance of these two species is either a signature of a young gas or, more likely, a dynamically excited gas. In fact, we find that for some of the models in which $\mathrm{HNCO}$ and/or $\mathrm{CH}_{3} \mathrm{OH}$ are very low before or at $10^{6} \mathrm{yr}$, their abundance is well enhanced at earlier times.

\section{Radiative transfer calculations: Intensities and intensities ratios}

We estimated the theoretical integrated intensity for the first 10 rotational lines of $\mathrm{CO}, \mathrm{HCN}, \mathrm{HCO}^{+}$, and $\mathrm{CS}$, and for selected lines of $\mathrm{SiO}, \mathrm{HNC}, \mathrm{SO}, \mathrm{CH}_{3} \mathrm{OH}$, and $\mathrm{HNCO}$, where the line selection is observationally motivated. The intensities were calculated using the radiative transfer code RADEX (van der Tak et al. 2007) from the grid of models in Tables A.2A.5, and Table 1. The collisional rates used for the RADEX calculations were taken from the LAMBDA database (Schöier et al. 2005) and were computed with $\mathrm{H}_{2}$ as collisional partner, where available. We used a background temperature of $2.7 \mathrm{~K}$. The approach we take in this paper differs from the usual use of RADEX or any LVG modelling in external galaxies in that we do not produce a grid in order to fit a set of observations. Rather, we used the input from our chemical modelling to fix the threeparameter set $\left(n_{\mathrm{H}}, T_{\text {gas }}, N(X)\right)$. Hence the only two free parameters in RADEX are the linewidth and geometry. We take two representative linewidths of 50 and $100 \mathrm{~km} \mathrm{~s}^{-1}$, but as the results do not affect our main conclusions we only present the $100 \mathrm{~km} \mathrm{~s}^{-1}$ models. Although RADEX can be used with different geometries, for the purpose of these calculations we used spherical geometry. While with Tables A.2-A.5, and Table 1 such calculations can be repeated for more geometries, Krips et al. (2011) used all three geometries available to characterize the dense gas in the AGN dominated nucleus of NGC 1068 but did not find significant differences in their fitting.

Also, estimating theoretical intensities for the shock models using RADEX falls well short of a proper radiative transfer calculation since the gas temperatures and densities vary quickly with the passage of the shock, and so do the abundances; hence one must be aware that these line intensities represent a gas that has been shocked (by a single or multiple shock episodes) but that has cooled back down to its "equilibrium" temperature (in this case $100 \mathrm{~K}$ ). Clearly a large variation of the parameter space can be performed when modelling shocked gas, which goes beyond the scope of this paper.

The results of our calculations are tabulated in Tables 2 and 3 , for the most commonly observed species, namely $\mathrm{CO}$, $\mathrm{HCO}^{+}, \mathrm{HCN}$ and CS; in Table 2 we list chemical abundances as obtained at $10^{7} \mathrm{yr}$ or at the last time step in the case of the shock models and in Table 3 we list chemical abundances as obtained at $10^{6} \mathrm{yr}$ for the models in which chemical equilibrium was not reached at this time. In Tables 5-11 we tabulate selected line intensities for $\mathrm{SiO}, \mathrm{SO}, \mathrm{HNC}$, and $\mathrm{HNCO}$. Because of the large number of $\mathrm{CH}_{3} \mathrm{OH}$ transitions observed as $k$ bands, we chose not to tabulate its line intensities (which can however be easily derived from the column densities tables). For some models, at $10^{6} \mathrm{yr}$, the RADEX calculation of the HCN does not converge; what these models have in common, at 1 million years, is that the $\mathrm{HCN}$ column density is very high and the gas is either very dense $\left(10^{6} \mathrm{~cm}^{-3}\right)$ and/or very hot $(200 \mathrm{~K})$. The issue of nonconvergence is most likely due to high-opacity effects, and in these cases Monte Carlo techniques combined with an accelerated lambda convergence method have been shown to perform better (e.g. Mills et al. 2013).
What it is often used in molecular studies in external galaxies are molecular line ratios. In Table 4 we tabulate the $\mathrm{HCN} / \mathrm{HCO}^{+}$ratios for each transition; we deliberately approximate to its integer value as it is seldom that one can claim a better accuracy from observational values. It is clear even from a quick glance that the often used ratios, for example the $\mathrm{HCN}(4-$ $3) / \mathrm{HCO}^{+}(4-3)$ or the $\mathrm{HCN}(1-0) / \mathrm{HCO}^{+}(1-0)$, are the same for several models within the accuracy assumed. While this is unavoidable, by approaching the interpretation of the observations with an a priori method such as presented here, one may, at least, be able to constrain this degeneracy. As a practical example, let us consider the $\mathrm{HCN} / \mathrm{HCO}^{+}$line ratios, which we plot in Fig. 1 for four different transitions. These ratios are routinely used as tracers of AGNs because they are found to be significantly higher in AGN when compared to those found in starbursts (e.g. Krips et al. 2008). Model 1 has the same $\mathrm{HCN} / \mathrm{HCO}^{+}(1-0)$ and similar $\mathrm{HCN} / \mathrm{HCO}^{+}(2-1)$ ratio to Model 7 but all the other ratios are very different. These two models differ substantially only in gas density, which is a physical characteristic that the lower transition ratios could not have traced. Figure 1 shows how a relatively standard grid of chemical models can lead to large ranges for the $\mathrm{HCN}(1-0) / \mathrm{HCO}^{+}$ratio of key transitions. This large range of ratios is consistent with the fact that, as pointed out in Krips et al. (2008), among others, differences in the intensity ratios may arise due to different densities, temperatures, radiation fields, and evolutionary state of activity. However, as long as more than two transitions per molecules are observed, degeneracies can indeed be broken, at least within the parameter space explored here.

The approach we outline here is not proposed as a substitute for the more canonical use of LVG modelling but as a complementary analysis. In particular one could use tables, such as those presented in this study, to determine a priori whether similar line intensities arise from very different physical conditions and viceversa. This may be of particular importance in light of the results from Tunnard \& Greve (2016) who find that LVG models struggle to recover any parameter better than to within half a dex and they find no evidence of systemic offsets. While these Tables are not exhaustive we hope that they can be used to track the origin of multiple solutions.

\section{Characterizing the dense gas in nearby galaxies}

In this section we provide examples of how one can use theoretically determined molecular intensities and ratios to interpret observations. We refer to two commonly observed nearby galaxies as our test beds and we compare an arbitrary selection of singledish observations for one of the galaxies, and interferometric observations for the second galaxy. What is presented here is not a full model of any of these two galaxies. In order to do that a much more comprehensive set of observations, together with a larger grid of models, ought to be used.

\section{1. $M 82$}

M 82 (at a distance of $3.5 \mathrm{Mpc}$ ) is one of the most studied nearby starburst galaxies. Among the many works, we chose, as an example, the observations presented in Aladro et al. (2011) who performed a line survey of the north-east (NE) lobe of M 82 with the single dish IRAM $30 \mathrm{~m}$ telescope. The spatial resolution obtained ranged from 158 to $333 \mathrm{kpc}$.

Of the molecular transitions they observed, we first compared CS (3-2), CS (5-4), and the $\mathrm{HCO}^{+}(3-2)$. We directly compared the integrated intensities (Table A.1 in Aladro et al. 2011) 
S. Viti: Molecular transitions as probes of the physical conditions of extragalactic environments
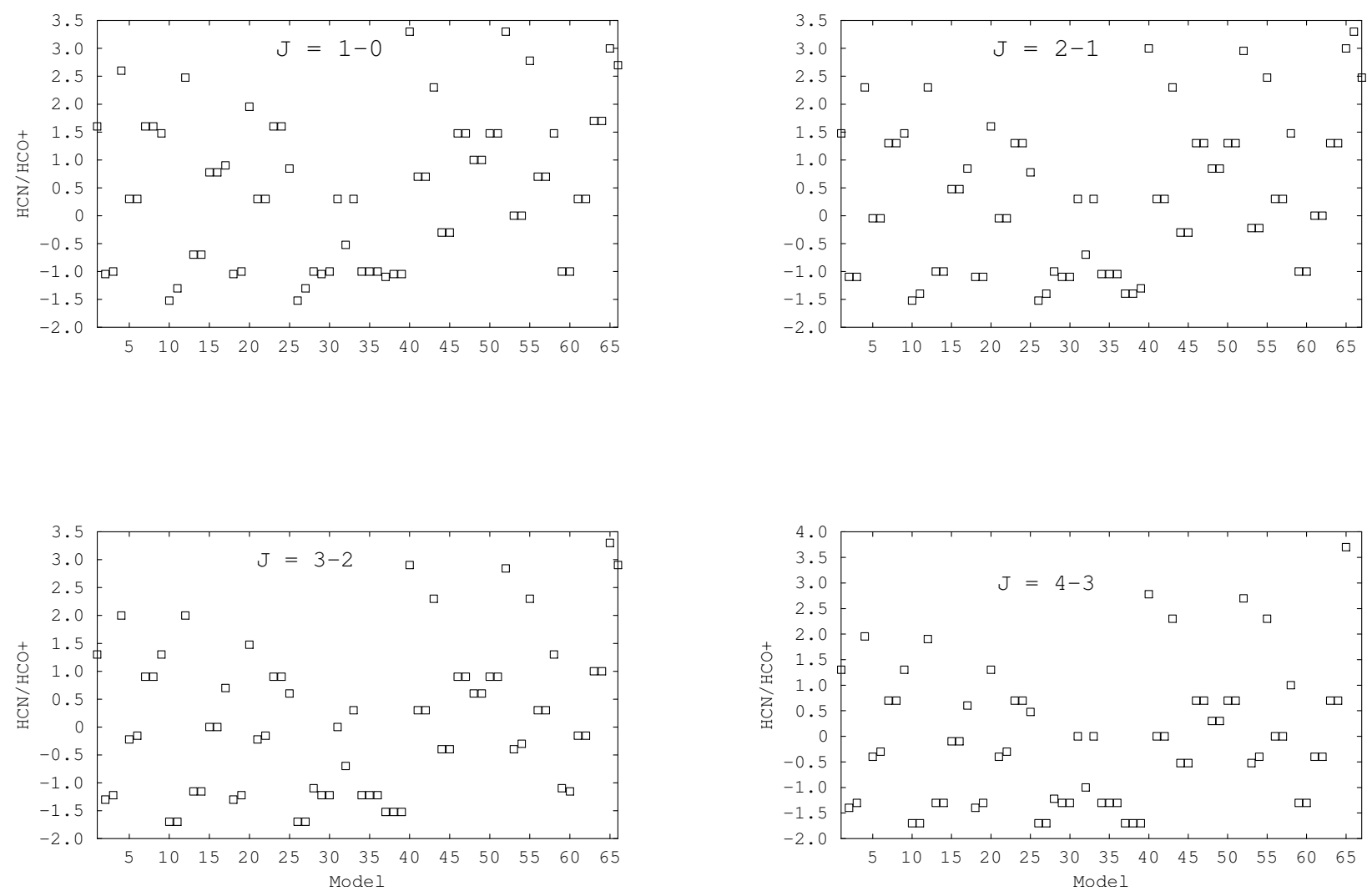

Fig. 1. Theoretical $\log$ line ratio $\mathrm{HCN} / \mathrm{HCO}^{+}$up to $J=4-3$ for each of the chemical model.

by making the crude assumption that the emission region fills the beam. In fact, according to Aladro et al. (2011), and based on previous interferometric observations, the emission region should be within a factor of $1-1.5$ of the beam size, hence our assumption is justified. However, substructures likely still exist within the beam and hence the observed line temperatures may be in fact smaller than the actual temperatures. The CS (3-2), observed to have an integrated line intensity of $\sim 11.5 \mathrm{~K} \mathrm{~km} \mathrm{~s}^{-1}$, is reasonably well matched by Models 8,24 , and to a lesser extent, 32. The first two models simulate very dense gas $\left(10^{6} \mathrm{~cm}^{-3}\right)$ and only differ in the strength of the radiation field, while the latter simulates a lower density but cosmic ray dominated gas. From this transition and single dish observations alone, we cannot distinguish between the two types of gas. On the other hand, for the CS (5-4) transition with an observed integrated line intensity of $\sim 4.8 \mathrm{~K} \mathrm{~km} \mathrm{~s}^{-1}$, the closest (but not a good fit) match is achieved by Models 49, 7 and 23; these all have a very high density gas but differ from those matching the lower $J$ transition either in visual extinction or temperature. The $\mathrm{HCO}^{+}(3-2)$ transition, observed to have an intensity of $\sim 4.2 \mathrm{~K} \mathrm{~km} \mathrm{~s}^{-1}$, is instead best matched by Model 44, simulating a low density $\left(10^{4} \mathrm{~cm}^{-3}\right)$, high temperature $(200 \mathrm{~K})$, and a low visual extinction gas, as one would expect as ions do not survive in very optically thick gas unless the ionization rates are enhanced. In fact, Model 44 best matches the $\mathrm{HCO}^{+}(3-2)$ transition at earlier times.

Of course, molecular ratios of lines are routinely used. This however makes the assumption that the two transitions arise from the same gas and that the individual column densities are both correct. For example, the $\mathrm{CS}(5-4) / \mathrm{CS}(3-2)$ ratio is best matched by Models 13, and then 6 and 22; none of these models match either of the individual line intensities.

Finally, comparing the CS LTE column density derived by Aladro et al. (2011) with our best matching models we find that all models matching the CS (3-2) can reproduce it, but, of the models that best match the CS (5-4) transition, Model 49, with the highest temperature and visual extinction, matches best the column density. The column density of $\mathrm{HCO}^{+}$is very well matched by the model that fits best its line intensity.

Aladro et al. (2011) also observed $\mathrm{SO}\left(4_{3}-3_{2}\right)$ and several methanol transitions. For the former its line intensity $\left(0.94 \mathrm{~K} \mathrm{~km} \mathrm{~s}^{-1}\right)$ is not well matched by any of our models, although its column density is close to that we derive for Model 33, highlighting the danger of just relying on column densities comparison alone (e.g. as in Viti et al. 2014). The observed column density for methanol, $\sim 10^{14} \mathrm{~cm}^{-2}$, is well matched by several models, of which all but one have a gas density of $10^{5} \mathrm{~cm}^{-3}$; interestingly all of the shock models over-predict its column density.

In summary, while a handful of single dish transitions are clearly not enough to characterize the multi-gas components of the nucleus of a galaxy, our ab initio method is able to certainly exclude ranges of densities, temperatures, and other physical parameters that do not affect the observed gas. It is clear that single dish observations cannot be interpreted by assuming different species and transitions as unique tracers of a particular environment. However this qualitative comparison already seems to indicate that while the CS (3-2) transition traces very dense gas at $\sim 100 \mathrm{~K}$, it is likely that the CS (5-4) traces a hotter component and the $\mathrm{HCO}^{+}$traces a hot, possibly young, gas, at a lower density. Comparisons with interferometric observations will offer stringent tests to our method, as outlined by the example below.

\subsection{NGC 253}

Again, as for the case of M82, here we simply perform an exercise to see how a priori modelling can help constrain some of the characteristics of the dense gas in another commonly studied galaxy. Meier et al. (2015) spatially resolved several molecular 
species in the central kiloparsec of NGC 253 (at a distance of $\sim 350 \mathrm{pc}$ ) with ALMA at a resolution of $\sim 50 \mathrm{pc}$. They identified 27 molecular lines in 10 individual locations. Meier et al. (2015) performed a detailed analysis highlighting the rich chemistry in the central zone of this galaxy. They reported the integrated line intensities, derived column densities, and LVG analyses. Their Fig. 5 shows an $\mathrm{HCN} / \mathrm{HCO}^{+}$ratio as a function of position going from $\sim 0.8$ to 1.6. Looking at Table 4 we find that this range is hard to match with any model and in fact those that are close to their best fit LVG model for the inner nuclear disk (positions 57 ), i.e at a gas density of $n_{\mathrm{H}}=10^{4.5-5.0} \mathrm{~cm}^{-3}$ and a gas temperature of $T=60-120 \mathrm{~K}$, all lead to a much higher $\mathrm{HCN}(1-$ $0) / \mathrm{HCO}^{+} 1-0$ ratio. This is consistent with Meier et al. (2015) conclusions that the main isotopologue $\mathrm{HCN} / \mathrm{HCO}^{+}$line ratios cannot alone be used as a unique diagnostic for starburst or AGN dominated galaxies, in this case probably owing to their high opacities. On the other hand, if we look at the individual theoretical intensities from Tables 2 and 3, we find that a good match for the HCN(1-0) transition can be obtained with Models 46 and 61 $\left(10^{5}-10^{6} \mathrm{~cm}^{-3}, 50-200 \mathrm{~K}\right)$ but only at early times, i.e. the gas does not seem to be in equilibrium. Interestingly, the gas traced by this transition does not seem to be affected by enhanced cosmic ray ionization rates. The $\mathrm{HCO}^{+}(1-0)$ transition in the same nuclear region can be matched by Models 27, 39, and 54, differing in physical characteristics but all sharing the commonality that the cosmic ray ionization rate is higher than standard; more importantly the gas is at equilibrium, implying that the gas that $\mathrm{HCO}^{+}$is tracing is not the same as that traced by $\mathrm{HCN}(1-0)$, which is another possible reason why their ratio is a poor indicator in this occasion.

Meier et al. (2015) also observes typical shock tracers: $\mathrm{SiO}$ and $\mathrm{HNCO}$. Making the assumption that $\mathrm{SiO}$ traces shocks they interpret the $\mathrm{SiO} / \mathrm{HCN}$ line ratio as an indicator of gas that is experiencing shocks. Yet, their $\mathrm{SiO}$ abundance, $\sim 10^{-9}$, which depending on the visual extinction gives column densities of $2-8 \times 10^{13} \mathrm{~cm}^{-2}$, is not matched by our shock models but rather by models that have an enhanced cosmic ray ionization rate (Models 33, 36, 39, see Table 5). On the other hand, HNCO can be matched by several models, including a shock model (Model 67). Hence, in this particular case, it seems that $\mathrm{SiO}$ can be used as a tracer of enhanced cosmic ray ionization gas.

Speculating further on the physical characteristics of each galaxy is beyond the scope of this paper but it is clear that a comparison of several transitions and species with large grids of chemical models may lead to a more complete picture than simply comparing molecular line ratios, especially if both column densities and individual line intensities, with appropriate corrections based on the filling factor of each observation, are taken into consideration.

\section{Conclusions}

We present a grid of chemical models and provide the abundances, abundance ratios, and column densities of the some of the most common tracers for a large parameter space in gas density, temperature, cosmic ray ionization rate, and radiation field (Tables A.1-A.5, and Table 1). The theoretical abundances are then used as inputs to a radiative transfer code to derive the theoretical intensities for the parameter space investigated by the chemical models (Tables 2, 3,5-11). We finally provide examples of comparisons of observations from two galaxies with our theoretical line intensities. Our main conclusions, which are only valid within the parameter space of chemical models that we ran here, are as follows: (i) time dependent effects on chemical abundances are only important for models in which the cosmic ray ionization rate is close to the galactic ionization rate. However the most affected species are, in fact, those most used to trace dense gas (HCN and CS). (ii) Line intensities ratios, such as $\mathrm{HCN}(1-0) / \mathrm{HCO}^{+}(1-0)$, are not unique across the grid, leading to clear degeneracies in any RADEX solution. (iii) Individual species can be unique tracers of a particular energetic process, but only within specific physical conditions. (iv) A brief analysis of molecular observations from two galaxies have shown how only relying on molecular line ratios can lead to erroneous conclusions on the physical conditions of the emitting gas and how therefore individual intensities, as well as column densities or fractional abundances, need to be taken into consideration.

Acknowledgements. The author acknowledges the AAO's Distinguished Visitor Scheme for their financial support during her visit at the AAO. The author thanks Dr. T. Greve and Dr. I. Jimenez-Serra for invaluable comments on the manuscript, and the anonymous referee for constructive comments that improved the original version of the paper.

\section{References}

Aladro, R., Martin, S., Martin-Pintado, J., et al. 2011, A\&A, 535, A84

Aladro, R., Viti, S., Bayet, E., et al. 2013, A\&A, 549, A39

Bayet, E., Viti, S., Williams, D. A., \& Rawlings, J. M. C. 2008, ApJ, 676, 978

Bayet, E., Viti, S., Williams, D. A., Rawlings, J. M. C., \& Bell, T. 2009, ApJ, 696, 1466

Bayet, E., Williams, D. A., Hartquist, T. W., \& Viti, S. 2011, MNRAS, 414, 627

Dyson, J. E., \& Williams, D. A. 1997, The Physics of the Interstellar Medium (Taylor \& Francis)

Green, C.-E., Cunningham, M. R., Green, J. A., et al. 2016, MNRAS, 457, 2470

Holdship, J., Viti, S., Jiménez-Serra, I., Makrymallis, A., \& Priestley, F. 2017, AJ, 154, 38

Jimenez-Serra, I., Caselli, P., Martín-Pintado, J., \& Hartquist, T. W. 2008, A\&A, 482, 549

Kazandjian, M. V., Meijerink, R., Pelupessy, I., Israel, F. P., \& Spaans, M. 2012, A\&A, 542, A65

Kazandjian, M. V., Meijerink, R., Pelupessy, I., Israel, F. P., \& Spaans, M. 2015, A\&A, 574, A127

Kohno, K., Matsushita, S., Vila-Vilaró, B., et al. 2001, in The Central Kiloparsec of Starbursts and AGN: The La Palma Connection, ASP Conf. Ser., 249, 672 Kohno, K., Tomoka, T., Takeshi, O., et al. 2005, PASP, 57, 147

Krips, M., Neri, R., García-Burillo, S., et al. 2008, ApJ, 677, 262

Krips, M., Martín, S., Eckart, A., et al. 2011, ApJ, 736, 37

Martín, S., Martín-Pintado, J., \& Mauersberger, R. 2009, ApJ, 694, 610

McElroy, D., Walsh, C., Markwick, A. J., et al. 2013, A\&A, 550, A36

Meier, D. S., Walter, F., Bolatto, A. D., et al. 2015, ApJ, 801, 63

Meijerink, R., Spaans, M., Loenen, A. F., \& van der Werf, P. P. 2011, A\&A, 525, A119

Meijerink, R., Spaans, M., Kamp, I., et al. 2013, J. Phys. Chem. A, 117, 9593

Mills, E. A. C., Güsten, R., Requena-Torres, M. A., \& Morris, M. R. 2013, ApJ, 779,47

Privon, G. C., Herrero-Illana, R., Evans, A. S., et al. 2015, ApJ, 814, 39

Schöier, F. L., van der Tak, F. F. S., van Dishoeck, E. F., \& Black, J. H. 2005, A\&A, 432, 369

Takano, S., Naomasa, N., \& Kentarou, K. 2002, PASP, 54, 195

Tan, B.-K., Leech, J., Rigopoulou, D., et al. 2013, MNRAS, 436, 921

Tunnard, R., \& Greve, T. R. 2016, ApJ, 819, 161

Usero, A., García-Burillo, S., Fuente, A., Martín-Pintado, J., \& Rodríguez-Fernández, N. J. 2004, A\&A, 419, 897

van der Tak, F. F. S., Black, J. H., Schöier, F. L., Jansen, D. J., \& van Dishoeck, E. F. 2007 , A\&A, 468, 627

van der Werf, P. P., Isaak, K. G., Meijerink, R., et al. 2010, A\&A, 518, L42

Viti, S. 2015, in IAU General Assembly, Meeting \#29, 2255280

Viti, S., Collings, M. P., Dever, J. W., McCoustra, M. R. S., \& Williams, D. A. 2004, MNRAS, 354, 1141

Viti, S., Jimenez-Serra, I., Yates, J. A., et al. 2011, ApJ, 740, L3

Viti, S., García-Burillo, S., Fuente, A., et al. 2014, A\&A, 570, A28

Wakelam, V., Loison, J.-C., Herbst, E., et al. 2015, ApJS, 217, 20

Williams, D. A., \& Viti, S. 2014, Observational Molecular Astronomy (Cambriudge University Press), 200

Zhao, Y., Lu, N., Xu, C. K., et al. 2016, ApJ, 820, 118 
S. Viti: Molecular transitions as probes of the physical conditions of extragalactic environments

\section{Appendix A: Tables}

Table A.1. Grid of chemical models and molecular abundances of selected species. $a(b)$ stands for $a \times 10^{b}$.

\begin{tabular}{|c|c|c|c|c|c|c|c|c|c|}
\hline$M$ & $\begin{array}{l}\zeta \\
\zeta_{0} \\
\end{array}$ & $\begin{array}{c}\chi \\
\text { Draine } \\
\end{array}$ & $\begin{array}{l}T \\
\mathrm{~K} \\
\end{array}$ & $\begin{array}{c}n_{\mathrm{H}} \\
\mathrm{cm}^{-3}\end{array}$ & $\begin{array}{c}A_{V} \\
\text { mag }\end{array}$ & $X(\mathrm{CO})$ & $X\left(\mathrm{HCO}^{+}\right)$ & $X(\mathrm{HCN})$ & $X(\mathrm{CS})$ \\
\hline 1 & 1 & 1 & 100 & $10^{4}$ & 1 & $2.6(-05)$ & $4.3(-11)$ & $6.9(-09)$ & $4.0(-08)$ \\
\hline 2 & 1 & 1 & 100 & $10^{4}$ & 10 & $2.7(-04)$ & $1.7(-09)$ & $9.1(-08)$ & $2.0(-07)$ \\
\hline 3 & 1 & 1 & 100 & $10^{4}$ & 50 & $2.7(-04)$ & $1.7(-09)$ & $8.4(-08)$ & $2.0(-07)$ \\
\hline 4 & 1 & 1 & 100 & $10^{5}$ & 1 & $8.0(-05)$ & $6.3(-11)$ & $6.4(-08)$ & $3.4(-08)$ \\
\hline 5 & 1 & 1 & 100 & $10^{5}$ & 10 & $2.7(-04)$ & $2.9(-10)$ & $5.0(-08)$ & $4.1(-08)$ \\
\hline 6 & 1 & 1 & 100 & $10^{5}$ & 50 & $2.7(-04)$ & $2.9(-10)$ & $5.0(-08)$ & $4.1(-08)$ \\
\hline 7 & 1 & 1 & 100 & $10^{6}$ & 10 & $2.7(-04)$ & $6.6(-11)$ & $5.5(-08)$ & $1.0(-09)$ \\
\hline 8 & 1 & 1 & 100 & $10^{6}$ & 50 & $2.7(-04)$ & $6.6(-11)$ & $5.5(-08)$ & $1.0(-09)$ \\
\hline 9 & 10 & 1 & 100 & $10^{4}$ & 1 & $2.5(-05)$ & $4.8(-11)$ & $6.8(-09)$ & $3.8(-08)$ \\
\hline 10 & 10 & 1 & 100 & $10^{4}$ & 10 & $2.7(-04)$ & $9.7(-09)$ & $1.2(-07)$ & $2.8(-07)$ \\
\hline 11 & 10 & 1 & 100 & $10^{4}$ & 50 & $2.7(-04)$ & $9.7(-09)$ & $1.2(-07)$ & $2.8(-07)$ \\
\hline 12 & 10 & 1 & 100 & $10^{5}$ & 1 & $8.0(-05)$ & $6.8(-11)$ & $6.1(-08)$ & $3.4(-08)$ \\
\hline 13 & 10 & 1 & 100 & $10^{5}$ & 10 & $2.7(-04)$ & $1.7(-09)$ & $9.7(-08)$ & $2.0(-07)$ \\
\hline 14 & 10 & 1 & 100 & $10^{5}$ & 50 & $2.7(-04)$ & $1.7(-09)$ & $9.7(-08)$ & $2.0(-07)$ \\
\hline 15 & 10 & 1 & 100 & $10^{6}$ & 10 & $2.7(-04)$ & $2.9(-10)$ & $5.0(-08)$ & $4.1(-08)$ \\
\hline 16 & 10 & 1 & 100 & $10^{6}$ & 50 & $2.7(-04)$ & $2.9(-10)$ & $5.1(-08)$ & $4.1(-08)$ \\
\hline 17 & 1 & 10 & 100 & $10^{4}$ & 1 & $2.8(-06)$ & $1.7(-11)$ & $5.6(-10)$ & $5.6(-09)$ \\
\hline 18 & 1 & 10 & 100 & $10^{4}$ & 10 & $2.7(-04)$ & $1.7(-09)$ & $9.1(-08)$ & $2.0(-07)$ \\
\hline 19 & 1 & 10 & 100 & $10^{4}$ & 50 & $2.7(-04)$ & $1.7(-09)$ & $8.4(-08)$ & $2.0(-07)$ \\
\hline 20 & 1 & 10 & 100 & $10^{5}$ & 1 & $2.6(-05)$ & $4.3(-11)$ & $6.9(-09)$ & $4.0(-08)$ \\
\hline 21 & 1 & 10 & 100 & $10^{5}$ & 10 & $2.7(-04)$ & $2.9(-10)$ & $5.0(-08)$ & $4.1(-08)$ \\
\hline 22 & 1 & 10 & 100 & $10^{5}$ & 50 & $2.7(-04)$ & $2.9(-10)$ & $5.0(-08)$ & $4.1(-08)$ \\
\hline 23 & 1 & 10 & 100 & $10^{6}$ & 10 & $2.7(-04)$ & $6.6(-11)$ & $5.5(-08)$ & $1.0(-09)$ \\
\hline 24 & 1 & 10 & 100 & $10^{6}$ & 50 & $2.7(-04)$ & $6.6(-11)$ & $5.5(-08)$ & $1.0(-09)$ \\
\hline 25 & 10 & 10 & 100 & $10^{4}$ & 1 & $2.8(-06)$ & $2.0(-11)$ & $5.6(-10)$ & $5.5(-09)$ \\
\hline 26 & 10 & 10 & 100 & $10^{4}$ & 10 & $2.7(-04)$ & $9.7(-09)$ & $1.2(-07)$ & $2.8(-07)$ \\
\hline 27 & 10 & 10 & 100 & $10^{4}$ & 50 & $2.7(-04)$ & $9.7(-09)$ & $1.2(-07)$ & $2.8(-07)$ \\
\hline 28 & 1 & 500 & 100 & $10^{4}$ & 1 & $2.5(-09)$ & $1.7(-12)$ & $1.0(-12)$ & $6.8(-12)$ \\
\hline 29 & 1 & 500 & 100 & $10^{4}$ & 10 & $2.7(-04)$ & $1.6(-09)$ & $9.6(-08)$ & $1.9(-07)$ \\
\hline 30 & 1 & 500 & 100 & $10^{4}$ & 50 & $2.7(-04)$ & $1.7(-09)$ & $8.4(-08)$ & $2.0(-07)$ \\
\hline 31 & 500 & 1 & 100 & $10^{4}$ & 1 & $1.2(-05)$ & $3.4(-10)$ & $2.8(-09)$ & $6.1(-09)$ \\
\hline 32 & 500 & 1 & 100 & $10^{4}$ & 10 & $2.5(-05)$ & $5.7(-10)$ & $4.6(-09)$ & $7.9(-09)$ \\
\hline 33 & 500 & 1 & 100 & $10^{4}$ & 50 & $2.5(-05)$ & $5.7(-10)$ & $4.6(-09)$ & $7.9(-09)$ \\
\hline 34 & 5000 & 1 & 100 & $10^{4}$ & 1 & $1.7(-05)$ & $2.2(-09)$ & $9.8(-10)$ & $1.3(-10)$ \\
\hline 35 & 5000 & 1 & 100 & $10^{4}$ & 10 & $1.9(-05)$ & $2.3(-09)$ & $1.1(-09)$ & $1.4(-10)$ \\
\hline 36 & 5000 & 1 & 100 & $10^{4}$ & 50 & $1.9(-05)$ & $2.3(-09)$ & $1.1(-09)$ & $1.4(-10)$ \\
\hline 37 & $10^{5}$ & 1 & 100 & $10^{5}$ & 1 & $1.8(-05)$ & $2.9(-09)$ & $4.5(-10)$ & $2.1(-11)$ \\
\hline 38 & $10^{5}$ & 1 & 100 & $10^{5}$ & 10 & $1.7(-05)$ & $2.8(-09)$ & $4.5(-10)$ & $2.1(-11)$ \\
\hline 39 & $10^{5}$ & 1 & 100 & $10^{5}$ & 50 & $1.7(-05)$ & $2.8(-09)$ & $4.5(-10)$ & $2.1(-11)$ \\
\hline 40 & 1 & 1 & 200 & $10^{5}$ & 1 & $6.8(-05)$ & $3.5(-11)$ & $8.2(-08)$ & $6.4(-09)$ \\
\hline 41 & 1 & 1 & 200 & $10^{5}$ & 10 & $2.7(-04)$ & $3.9(-10)$ & $2.9(-08)$ & $2.6(-09)$ \\
\hline 42 & 1 & 1 & 200 & $10^{5}$ & 50 & $2.7(-04)$ & $3.9(-10)$ & $2.9(-08)$ & $2.6(-09)$ \\
\hline 43 & 1 & 1 & 200 & $10^{4}$ & 1 & $1.8(-05)$ & $2.3(-11)$ & $1.7(-08)$ & $1.8(-08)$ \\
\hline 44 & 1 & 1 & 200 & $10^{4}$ & 10 & $2.7(-04)$ & $2.5(-09)$ & $3.0(-07)$ & 7.6(-09) \\
\hline 45 & 1 & 1 & 200 & $10^{4}$ & 50 & $2.7(-04)$ & $2.5(-09)$ & $2.8(-07)$ & $7.6(-09)$ \\
\hline 46 & 1 & 1 & 200 & $10^{6}$ & 10 & $2.6(-04)$ & $8.3(-11)$ & $1.1(-08)$ & $7.7(-11)$ \\
\hline 47 & 1 & 1 & 200 & $10^{6}$ & 50 & $2.7(-04)$ & $8.3(-11)$ & $1.1(-08)$ & $7.6(-11)$ \\
\hline 48 & 10 & 1 & 200 & $10^{6}$ & 10 & $2.6(-04)$ & $4.0(-10)$ & $2.9(-08)$ & $2.6(-09)$ \\
\hline 49 & 10 & 1 & 200 & $10^{6}$ & 50 & $2.7(-04)$ & $3.9(-10)$ & $3.0(-08)$ & $2.6(-09)$ \\
\hline 50 & 1 & 10 & 200 & $10^{6}$ & 10 & $2.6(-04)$ & $8.3(-11)$ & $1.1(-08)$ & $7.7(-11)$ \\
\hline 51 & 1 & 10 & 200 & $10^{6}$ & 50 & $2.7(-04)$ & $8.3(-11)$ & $1.1(-08)$ & $7.6(-11)$ \\
\hline 52 & 10 & 1 & 200 & $10^{5}$ & 1 & $6.9(-05)$ & $4.0(-11)$ & $8.1(-08)$ & $6.7(-09)$ \\
\hline 53 & 10 & 1 & 200 & $10^{5}$ & 10 & $2.7(-04)$ & $2.5(-09)$ & $3.1(-07)$ & 7.6(-09) \\
\hline 54 & 10 & 1 & 200 & $10^{5}$ & 50 & $2.7(-04)$ & $2.5(-09)$ & $3.1(-07)$ & $7.5(-09)$ \\
\hline 55 & 1 & 10 & 200 & $10^{5}$ & 1 & $1.8(-05)$ & $2.2(-11)$ & $1.8(-08)$ & $1.8(-08)$ \\
\hline 56 & 1 & 10 & 200 & $10^{5}$ & 10 & $2.7(-04)$ & $3.9(-10)$ & $2.9(-08)$ & $2.6(-09)$ \\
\hline 57 & 1 & 10 & 200 & $10^{5}$ & 50 & $2.7(-04)$ & $3.9(-10)$ & $2.9(-08)$ & $2.6(-09)$ \\
\hline 58 & 1 & 1 & 50 & $10^{4}$ & 1 & $3.5(-05)$ & $4.4(-11)$ & $6.4(-09)$ & $5.1(-08)$ \\
\hline 59 & 1 & 1 & 50 & $10^{4}$ & 10 & $2.7(-04)$ & $1.1(-09)$ & $5.4(-08)$ & $1.3(-06)$ \\
\hline 60 & 1 & 1 & 50 & $10^{4}$ & 50 & $2.7(-04$ & $1.1(-09)$ & $4.8(-08)$ & $1.4(-06)$ \\
\hline 61 & 1 & 1 & 50 & $10^{5}$ & 10 & $2.7(-04)$ & $2.3(-10)$ & $4.3(-08)$ & $4.9(-07)$ \\
\hline 62 & 1 & 1 & 50 & $10^{5}$ & 50 & $2.7(-04)$ & $2.3(-10)$ & $4.3(-08)$ & $4.9(-07)$ \\
\hline 63 & 1 & 1 & 50 & $10^{6}$ & 10 & $2.7(-04)$ & $5.6(-11)$ & $5.6(-08)$ & $3.6(-08)$ \\
\hline 64 & 1 & 1 & 50 & $10^{6}$ & 50 & $2.7(-04)$ & $5.6(-11)$ & $5.6(-08)$ & $3.6(-08)$ \\
\hline 65 & 1 & 1 & shock & $10^{4}$ & 10 & $1.6(-04)$ & $5.1(-12)$ & $1.3(-05)$ & $7.7(-09)$ \\
\hline 66 & 1 & 1 & shock & $10^{5}$ & 10 & $2.5(-04)$ & $1.7(-12)$ & $1.3(-06)$ & $5.3(-08)$ \\
\hline 67 & 1 & 1 & shock & $10^{6}$ & 10 & $2.3(-04)$ & $1.4(-13)$ & $1.2(-06)$ & $4.5(-09)$ \\
\hline
\end{tabular}


Table A.2. Grid of chemical models, molecular column densities and ratios of selected species. $a(b)$ stands for $a \times 10^{b}$.

\begin{tabular}{|c|c|c|c|c|c|c|c|c|c|c|c|c|c|}
\hline$M$ & $\begin{array}{l}\zeta \\
\zeta_{0}\end{array}$ & $\begin{array}{l}\chi \\
\text { Draine }\end{array}$ & $\begin{array}{l}T \\
\mathrm{~K} \\
\end{array}$ & $\begin{array}{l}n_{H} \\
\mathrm{~cm}^{-3}\end{array}$ & $\begin{array}{l}A_{V} \\
\text { mags }\end{array}$ & $\begin{array}{l}N(\mathrm{CO}) \\
\mathrm{cm}^{-2}\end{array}$ & $\begin{array}{l}N\left(\mathrm{HCO}^{+}\right) \\
\mathrm{cm}^{-2}\end{array}$ & $\begin{array}{l}N(\mathrm{HCN}) \\
\mathrm{cm}^{-2}\end{array}$ & $\begin{array}{l}N(\mathrm{CS}) \\
\mathrm{cm}^{-2}\end{array}$ & $\begin{array}{l}\mathrm{HCN} / \\
\mathrm{HCO}^{+}\end{array}$ & $\begin{array}{l}\mathrm{HCN} / \\
\mathrm{CO}\end{array}$ & $\begin{array}{l}\mathrm{HCO}^{+} / \\
\mathrm{CO}\end{array}$ & $\begin{array}{l}\mathrm{CS} / \\
\mathrm{CO}\end{array}$ \\
\hline 1 & 1 & 1 & 100 & $10^{4}$ & 1 & $2.3(16)$ & $3.0(10)$ & $1.1(13)$ & $6.4(13)$ & $3.7(02)$ & $4.8(-04)$ & $1.3(-06)$ & $2.8(-03)$ \\
\hline 2 & 1 & 1 & 100 & $10^{4}$ & 10 & $4.3(18)$ & $1.1(12)$ & $1.5(15)$ & $3.2(15)$ & $1.3(03)$ & $3.4(-04)$ & $2.6(-07)$ & $7.5(-04)$ \\
\hline 3 & 1 & 1 & 100 & $10^{4}$ & 50 & $2.1(19)$ & $5.7(12)$ & $6.7(15)$ & $1.6(16)$ & $1.2(03)$ & $3.2(-04)$ & $2.6(-07)$ & $7.5(-04)$ \\
\hline 4 & 1 & 1 & 100 & $10^{5}$ & 1 & $3.7(16)$ & $4.5(09)$ & $1.0(14)$ & $5.5(13)$ & $2.3(04)$ & $2.8(-03)$ & $1.2(-07)$ & $1.5(-03)$ \\
\hline 5 & 1 & 1 & 100 & $10^{5}$ & 10 & $4.3(18)$ & $4.5(11)$ & $8.1(14)$ & $6.6(14)$ & $1.8(03)$ & $1.9(-04)$ & $1.1(-07)$ & $1.5(-04)$ \\
\hline 6 & 1 & 1 & 100 & $10^{5}$ & 50 & $2.1(19)$ & $2.3(12)$ & $4.0(15)$ & $3.3(15)$ & $1.8(03)$ & $1.9(-04)$ & $1.1(-07)$ & $1.5(-04)$ \\
\hline 7 & 1 & 1 & 100 & $10^{6}$ & 10 & $4.2(18)$ & $1.1(11)$ & $8.8(14)$ & $1.6(13)$ & 7.8(03) & $2.1(-04)$ & $2.7(-08)$ & $4.0(-06)$ \\
\hline 8 & 1 & 1 & 100 & $10^{6}$ & 50 & $2.1(19)$ & $5.6(11)$ & $4.4(15)$ & $8.2(13)$ & 7.9(03) & $2.1(-04)$ & $2.7(-08)$ & $3.9(-06)$ \\
\hline 9 & 10 & 1 & 100 & $10^{4}$ & 1 & $2.2(16)$ & $3.5(10)$ & $1.1(13)$ & $6.1(13)$ & $3.1(02)$ & $4.9(-04)$ & $1.5(-06)$ & $2.7(-03)$ \\
\hline 10 & 10 & 1 & 100 & $10^{4}$ & 10 & $3.6(18)$ & $1.0(12)$ & $2.0(15)$ & $4.6(15)$ & $1.9(03)$ & $5.6(-04)$ & $2.9(-07)$ & $1.3(-03)$ \\
\hline 11 & 10 & 1 & 100 & $10^{4}$ & 50 & $1.8(19)$ & $5.2(12)$ & $9.9(15)$ & $2.3(16)$ & $1.9(03)$ & $5.6(-04)$ & $2.9(-07)$ & $1.3(-03)$ \\
\hline 12 & 10 & 1 & 100 & $10^{5}$ & 1 & $3.7(16)$ & $4.9(09)$ & $9.8(13)$ & $5.5(13)$ & $2.0(04)$ & $2.6(-03)$ & $1.3(-07)$ & $1.5(-03)$ \\
\hline 13 & 10 & 1 & 100 & $10^{5}$ & 10 & $4.3(18)$ & $1.1(12)$ & $1.6(15)$ & $3.2(15)$ & $1.4(03)$ & $3.6(-04)$ & $2.7(-07)$ & 7.4(-04) \\
\hline 14 & 10 & 1 & 100 & $10^{5}$ & 50 & $2.1(19)$ & $5.6(12)$ & $7.8(15)$ & $1.6(16)$ & $1.4(03)$ & $3.7(-04)$ & $2.6(-07)$ & 7.4(-04) \\
\hline 15 & 10 & 1 & 100 & $10^{6}$ & 10 & $4.3(18)$ & $4.6(11)$ & $8.1(14)$ & $6.5(14)$ & $1.8(03)$ & $1.9(-04)$ & $1.1(-07)$ & $1.5(-04)$ \\
\hline 16 & 10 & 1 & 100 & $10^{6}$ & 50 & $2.1(19)$ & $2.3(12)$ & $4.1(15)$ & $3.3(15)$ & $1.8(03)$ & $1.9(-04)$ & $1.1(-07)$ & $1.5(-04)$ \\
\hline 17 & 1 & 10 & 100 & $10^{4}$ & 1 & $3.5(15)$ & $1.8(10)$ & $8.9(11)$ & $9.0(12)$ & $4.8(01)$ & $2.5(-04)$ & $5.2(-06)$ & $2.5(-03)$ \\
\hline 18 & 1 & 10 & 100 & $10^{4}$ & 10 & $4.3(18)$ & $1.1(12)$ & $1.5(15)$ & $3.2(15)$ & $1.3(03)$ & $3.4(-04)$ & $2.6(-07)$ & 7.4(-04) \\
\hline 19 & 1 & 10 & 100 & $10^{4}$ & 50 & $2.1(19)$ & $5.7(12)$ & $6.7(15)$ & $1.6(16)$ & $1.2(03)$ & $3.2(-04)$ & $2.6(-07)$ & $7.5(-04)$ \\
\hline 20 & 1 & 10 & 100 & $10^{5}$ & 1 & $2.3(16)$ & $3.0(10)$ & $1.1(13)$ & $6.5(13)$ & $3.8(02)$ & $4.8(-04)$ & $1.3(-06)$ & $2.8(-03)$ \\
\hline 21 & 1 & 10 & 100 & $10^{5}$ & 10 & $4.3(18)$ & $4.5(11)$ & $8.0(14)$ & $6.6(14)$ & $1.8(03)$ & $1.9(-04)$ & $1.1(-07)$ & $1.5(-04)$ \\
\hline 22 & 1 & 10 & 100 & $10^{5}$ & 50 & $2.1(19)$ & $2.3(12)$ & $4.0(15)$ & $3.3(15)$ & $1.8(03)$ & $1.9(-04)$ & $1.1(-07)$ & $1.5(-04)$ \\
\hline 23 & 1 & 10 & 100 & $10^{6}$ & 10 & $4.2(18)$ & $1.1(11)$ & $8.8(14)$ & $1.6(13)$ & 7.8(03) & $2.1(-04)$ & $2.7(-08)$ & $4.0(-06)$ \\
\hline 24 & 1 & 10 & 100 & $10^{6}$ & 50 & 2.1(19) & $5.6(11)$ & $4.4(15)$ & $8.2(13)$ & $7.9(03)$ & $2.1(-04)$ & $2.7(-08)$ & $3.9(-06)$ \\
\hline 25 & 10 & 10 & 100 & $10^{4}$ & 1 & $3.5(15)$ & $2.1(10)$ & $8.9(11)$ & $8.8(12)$ & $4.2(01)$ & $2.6(-04)$ & $6.1(-06)$ & $2.5(-03)$ \\
\hline 26 & 10 & 10 & 100 & $10^{4}$ & 10 & $3.5(18)$ & $1.0(12)$ & $2.0(15)$ & $4.6(15)$ & $1.9(03)$ & $5.6(-04)$ & $2.9(-07)$ & $1.3(-03)$ \\
\hline 27 & 10 & 10 & 100 & $10^{4}$ & 50 & $1.8(19)$ & $5.2(12)$ & $9.9(15)$ & $2.3(16)$ & $1.9(03)$ & $5.6(-04)$ & $2.9(-07)$ & $1.3(-03)$ \\
\hline 28 & 1 & 500 & 100 & $10^{4}$ & 1 & $3.6(12)$ & $1.9(09)$ & $1.7(09)$ & $1.1(10)$ & $8.8(-01)$ & $4.6(-04)$ & $5.2(-04)$ & $3.0(-03)$ \\
\hline 29 & 1 & 500 & 100 & $10^{4}$ & 10 & $4.3(18)$ & $1.1(12)$ & $1.5(15)$ & $3.1(15)$ & $1.4(03)$ & $3.6(-04)$ & $2.6(-07)$ & 7.3(-04) \\
\hline 30 & 1 & 500 & 100 & $10^{4}$ & 50 & $2.1(19)$ & $5.7(12)$ & $6.7(15)$ & $1.6(16)$ & $1.2(03)$ & $3.2(-04)$ & $2.6(-07)$ & $7.5(-04)$ \\
\hline 31 & 500 & 1 & 100 & $10^{4}$ & 1 & $1.4(16)$ & $3.2(11)$ & $4.5(12)$ & $9.8(12)$ & $1.4(01)$ & $3.2(-04)$ & $2.3(-05)$ & $7.1(-04)$ \\
\hline 32 & 500 & 1 & 100 & $10^{4}$ & 10 & $2.3(17)$ & $3.7(12)$ & 7.4(13) & $1.3(14)$ & $2.0(01)$ & $3.2(-04)$ & $1.6(-05)$ & $5.5(-04)$ \\
\hline 33 & 500 & 1 & 100 & $10^{4}$ & 50 & $1.1(18)$ & $1.8(13)$ & $3.7(14)$ & $6.3(14)$ & $2.0(01)$ & $3.2(-04)$ & $1.6(-05)$ & $5.5(-04)$ \\
\hline 34 & 5000 & 1 & 100 & $10^{4}$ & 1 & $2.0(16)$ & $2.3(12)$ & $1.6(12)$ & 2.1(11) & $6.9(-01)$ & $8.0(-05)$ & $1.2(-04)$ & $1.1(-05)$ \\
\hline 35 & 5000 & 1 & 100 & $10^{4}$ & 10 & $2.1(17)$ & $2.4(13)$ & $1.7(13)$ & $2.3(12)$ & $7.2(-01)$ & $8.1(-05)$ & $1.1(-04)$ & $1.1(-05)$ \\
\hline 36 & 5000 & 1 & 100 & $10^{4}$ & 50 & $1.1(18)$ & $1.2(14)$ & $8.5(13)$ & $1.1(13)$ & $7.2(-01)$ & $8.1(-05)$ & $1.1(-04)$ & $1.1(-05)$ \\
\hline 37 & $10^{5}$ & 1 & 100 & $10^{5}$ & 1 & $2.0(16)$ & $3.1(12)$ & $7.3(11)$ & $3.3(10)$ & $2.4(-01)$ & $3.6(-05)$ & $1.5(-04)$ & $1.7(-06)$ \\
\hline 38 & $10^{5}$ & 1 & 100 & $10^{5}$ & 10 & $2.0(17)$ & $3.1(13)$ & $7.3(12)$ & $3.3(11)$ & $2.4(-01)$ & $3.7(-05)$ & $1.6(-04)$ & $1.7(-06)$ \\
\hline 39 & $10^{5}$ & 1 & 100 & $10^{5}$ & 50 & $9.7(17)$ & $1.5(14)$ & $3.6(13)$ & $1.7(12)$ & $2.4(-01)$ & $3.7(-05)$ & $1.6(-04)$ & $1.7(-06)$ \\
\hline 40 & 1 & 1 & 200 & $10^{5}$ & 1 & $3.8(16)$ & $5.3(09)$ & $1.3(14)$ & $1.0(13)$ & $2.5(04)$ & $3.4(-03)$ & $1.4(-07)$ & $2.7(-04)$ \\
\hline 41 & 1 & 1 & 200 & $10^{5}$ & 10 & $4.3(18)$ & $5.4(11)$ & $4.7(14)$ & $4.2(13)$ & $8.8(02)$ & $1.1(-04)$ & $1.3(-07)$ & $9.9(-06)$ \\
\hline 42 & 1 & 1 & 200 & $10^{5}$ & 50 & $2.1(19)$ & $2.7(12)$ & $2.3(15)$ & $2.1(14)$ & $8.7(02)$ & $1.1(-04)$ & $1.3(-07)$ & $9.9(-06)$ \\
\hline 43 & 1 & 1 & 200 & $10^{4}$ & 1 & $1.8(16)$ & $2.0(10)$ & $2.8(13)$ & $2.9(13)$ & $1.4(03)$ & $1.5(-03)$ & $1.1(-06)$ & $1.6(-03)$ \\
\hline 44 & 1 & 1 & 200 & $10^{4}$ & 10 & $4.2(18)$ & $1.4(12)$ & $4.8(15)$ & $1.2(14)$ & $3.5(03)$ & $1.1(-03)$ & $3.2(-07)$ & $2.9(-05)$ \\
\hline 45 & 1 & 1 & 200 & $10^{4}$ & 50 & $2.1(19)$ & $6.7(12)$ & $2.3(16)$ & 6.1(14) & $3.4(03)$ & $1.1(-03)$ & $3.2(-07)$ & $2.9(-05)$ \\
\hline 46 & 1 & 1 & 200 & $10^{6}$ & 10 & 4.1(18) & $1.4(11)$ & $1.8(14)$ & $1.2(12)$ & $1.3(03)$ & $4.3(-05)$ & $3.4(-08)$ & $3.0(-07)$ \\
\hline 47 & 1 & 1 & 200 & $10^{6}$ & 50 & $2.1(19)$ & $6.9(11)$ & $8.9(14)$ & $6.1(12)$ & $1.3(03)$ & $4.3(-05)$ & $3.4(-08)$ & $3.0(-07)$ \\
\hline 48 & 10 & 1 & 200 & $10^{6}$ & 10 & $4.3(18)$ & $5.4(11)$ & $4.7(14)$ & $4.2(13)$ & $8.7(02)$ & $1.1(-04)$ & $1.3(-07)$ & $9.8(-06)$ \\
\hline 49 & 10 & 1 & 200 & $10^{6}$ & 50 & 2.1(19) & $2.7(12)$ & $2.4(15)$ & $2.1(14)$ & $8.8(02)$ & $1.1(-04)$ & $1.3(-07)$ & $9.9(-06)$ \\
\hline 50 & 1 & 10 & 200 & $10^{6}$ & 10 & 4.1(18) & $1.4(11)$ & $1.8(14)$ & $1.2(12)$ & $1.3(03)$ & $4.3(-05)$ & $3.4(-08)$ & $3.0(-07)$ \\
\hline 51 & 1 & 10 & 200 & $10^{6}$ & 50 & $2.1(19)$ & $6.9(11)$ & $8.9(14)$ & $6.1(12)$ & $1.3(03)$ & $4.3(-05)$ & $3.4(-08)$ & $3.0(-07)$ \\
\hline 52 & 10 & 1 & 200 & $10^{5}$ & 1 & $3.9(16)$ & $6.0(09)$ & $1.3(14)$ & $1.1(13)$ & $2.2(04)$ & $3.3(-03)$ & $1.5(-07)$ & $2.7(-04)$ \\
\hline 53 & 10 & 1 & 200 & $10^{5}$ & 10 & $4.2(18)$ & $1.4(12)$ & $5.0(15)$ & $1.2(14)$ & $3.7(03)$ & $1.2(-03)$ & $3.2(-07)$ & $2.9(-05)$ \\
\hline 54 & 10 & 1 & 200 & $10^{5}$ & 50 & 2.1(19) & $6.7(12)$ & $2.5(16)$ & $6.0(14)$ & $3.7(03)$ & $1.2(-03)$ & $3.2(-07)$ & $2.9(-05)$ \\
\hline 55 & 1 & 10 & 200 & $10^{5}$ & 1 & $1.8(16)$ & $1.9(10)$ & $2.8(13)$ & $2.9(13)$ & $1.5(03)$ & $1.6(-03)$ & $1.0(-06)$ & $1.6(-03)$ \\
\hline 56 & 1 & 10 & 200 & $10^{5}$ & 10 & $4.3(18)$ & $5.4(11)$ & $4.7(14)$ & $4.2(13)$ & $8.8(02)$ & $1.1(-04)$ & $1.3(-07)$ & $9.9(-06)$ \\
\hline 57 & 1 & 10 & 200 & $10^{5}$ & 50 & $2.1(19)$ & $2.7(12)$ & $2.3(15)$ & $2.1(14)$ & $8.7(02)$ & $1.1(-04)$ & $1.3(-07)$ & $9.9(-06)$ \\
\hline 58 & 1 & 10 & 50 & $10^{4}$ & 1 & $2.6(16)$ & $2.2(10)$ & $1.0(13)$ & 8.1(13) & $4.6(02)$ & $3.9(-04)$ & $8.6(-07)$ & $3.1(-03)$ \\
\hline 59 & 1 & 10 & 50 & $10^{4}$ & 10 & $4.3(18)$ & $9.3(11)$ & $8.7(14)$ & $2.1(16)$ & $9.4(02)$ & $2.0(-04)$ & $2.2(07)$ & $5.0(03)$ \\
\hline 60 & 1 & 10 & 50 & $10^{4}$ & 50 & $2.1(19)$ & $4.6(12)$ & $3.9(15)$ & $1.1(17)$ & $8.4(02)$ & $1.8(-04)$ & $2.2(07)$ & $5.1(03)$ \\
\hline 61 & 1 & 1 & 50 & $10^{5}$ & 10 & $4.3(18)$ & $3.8(11)$ & $6.9(14)$ & $7.9(15)$ & $1.8(03)$ & $1.6(-04)$ & $8.9(-08)$ & $1.9(-03)$ \\
\hline 62 & 1 & 1 & 50 & $10^{5}$ & 50 & $2.1(19)$ & $1.9(12)$ & $3.4(15)$ & $4.0(16)$ & $1.8(03)$ & $1.6(-04)$ & $8.9(-08)$ & $1.9(-03)$ \\
\hline 63 & 1 & 1 & 50 & $10^{6}$ & 10 & $4.2(18)$ & $9.3(10)$ & $8.9(14)$ & $5.8(14)$ & $9.6(03)$ & $2.1(-04)$ & $2.2(-08)$ & $1.4(-04)$ \\
\hline 64 & 1 & 1 & 50 & $10^{6}$ & 50 & $2.1(19)$ & $4.6(11)$ & $4.5(15)$ & $2.9(15)$ & $9.7(03)$ & $2.1(-04)$ & $2.2(-08)$ & $1.4(-04)$ \\
\hline 65 & 1 & 1 & shock & $10^{4}$ & 10 & $3.6(20)$ & $6.8(12)$ & $3.8(19)$ & $2.3(16)$ & $5.6(06)$ & $1.1(-01)$ & $1.9(-08)$ & $6.2(-05)$ \\
\hline 66 & 1 & 1 & shock & $10^{5}$ & 10 & $8.2(20)$ & $4.5(12)$ & $4.5(18)$ & $1.8(17)$ & $9.9(05)$ & $5.5(-03)$ & $5.5(-09)$ & $2.2(-04)$ \\
\hline 67 & 1 & 1 & shock & $10^{6}$ & 10 & $8.9(20)$ & $5.3(11)$ & $4.5(18)$ & $1.7(16)$ & $8.4(06)$ & $5.0(-03)$ & $6.0(-10)$ & $1.9(-05)$ \\
\hline
\end{tabular}


S. Viti: Molecular transitions as probes of the physical conditions of extragalactic environments

Table A.3. Column densities at $10^{6} \mathrm{yr}$ for Models showing changes between 1 and 10 million years. $a(b)$ stands for $a \times 10^{b}$.

\begin{tabular}{|c|c|c|c|c|}
\hline$M$ & $N(\mathrm{CO})$ & $\begin{array}{r}N\left(\mathrm{HCO}^{+}\right) \\
\mathrm{cm} \\
\end{array}$ & $N(\mathrm{HCN})$ & $N(\mathrm{CS})$ \\
\hline 2 & $4.1(18)$ & $2.8(13)$ & $2.6(15)$ & $4.0(15)$ \\
\hline 3 & $2.0(19)$ & $1.3(14)$ & $7.3(15)$ & $1.0(16)$ \\
\hline 5 & $3.8(18)$ & $1.3(12)$ & $3.1(15)$ & $3.7(13)$ \\
\hline 6 & $1.4(19)$ & $1.6(12)$ & $4.8(16)$ & $1.0(14)$ \\
\hline 7 & $2.1(18)$ & $1.3(10)$ & $8.2(15)$ & $4.0(13)$ \\
\hline 8 & $1.5(19)$ & $3.2(11)$ & $2.8(15)$ & $1.2(14)$ \\
\hline 9 & $4.0(16)$ & $7.7(10)$ & $1.5(13)$ & $1.3(14)$ \\
\hline 18 & $4.1(18)$ & $2.8(13)$ & $2.6(15)$ & $4.0(15)$ \\
\hline 19 & $2.0(19)$ & $1.3(14)$ & 7.3(15) & $1.0(16)$ \\
\hline 21 & $3.8(18)$ & $1.3(12)$ & $3.1(15)$ & $4.0(13)$ \\
\hline 22 & $1.4(19)$ & $1.6(12)$ & $4.8(16)$ & $1.0(14)$ \\
\hline 23 & 2.1(18) & $1.3(10)$ & 8.1(15) & $3.9(13)$ \\
\hline 24 & $1.5(19)$ & $3.2(11)$ & $2.8(15)$ & $1.2(14)$ \\
\hline 29 & $4.1(18)$ & $2.8(13)$ & $2.5(15)$ & $4.2(15)$ \\
\hline 30 & $2.0(19)$ & $1.3(14)$ & $7.3(15)$ & $1.0(16)$ \\
\hline 41 & $3.9(18)$ & $1.3(12)$ & $9.6(15)$ & $7.5(13)$ \\
\hline 42 & $1.7(19)$ & $1.7(12)$ & $2.0(17)$ & $8.7(14)$ \\
\hline 44 & $4.1(18)$ & $3.8(13)$ & $4.7(15)$ & $2.2(14)$ \\
\hline 45 & $2.0(19)$ & $1.7(14)$ & $1.5(16)$ & $8.1(14)$ \\
\hline 46 & $2.6(18)$ & $1.6(10)$ & $1.8(16)$ & $1.4(15)$ \\
\hline 47 & $1.9(19)$ & $2.5(11)$ & $8.3(16)$ & $4.2(14)$ \\
\hline 50 & $2.6(18)$ & $1.6(10)$ & $1.7(16)$ & $1.5(15)$ \\
\hline 51 & $1.9(19)$ & $2.5(11)$ & $8.3(16)$ & $4.2(14)$ \\
\hline 56 & $3.9(18)$ & $1.3(12)$ & $9.5(15)$ & $7.4(13)$ \\
\hline 57 & $1.7(19)$ & $1.7(12)$ & $2.0(17)$ & $8.7(14)$ \\
\hline 58 & $5.7(16)$ & 7.1(10) & $1.4(13)$ & $2.3(14)$ \\
\hline 59 & $4.0(18)$ & 2.1(13) & 2.3(15) & $1.0(16)$ \\
\hline 60 & $2.0(19)$ & $9.5(13)$ & $6.7(15)$ & $1.6(16)$ \\
\hline 61 & $3.8(18)$ & $7.8(11)$ & $2.9(15)$ & $8.4(12)$ \\
\hline 62 & $1.6(19)$ & 9.1(11) & $4.0(16)$ & $2.7(13)$ \\
\hline 63 & $2.6(18)$ & 7.7(09) & $5.7(15)$ & $7.0(12)$ \\
\hline 64 & $1.7(19)$ & $1.7(11)$ & $1.6(15)$ & $2.0(13)$ \\
\hline
\end{tabular}

Table A.4. Column densities at $10^{7} \mathrm{yr}$ for other selected species. $a(b)$ stands for $a \times 10^{b}$.

\begin{tabular}{|c|c|c|c|}
\hline$M$ & $N(\mathrm{SiO})$ & $\begin{array}{c}N(\mathrm{HNC}) \\
\mathrm{cm}^{-2}\end{array}$ & $N(\mathrm{SO})$ \\
\hline 1 & $6.9(09)$ & $1.2(11)$ & $1.1(11)$ \\
\hline 2 & $5.2(15)$ & $5.0(12)$ & $1.4(17)$ \\
\hline 3 & $2.6(16)$ & $2.5(13)$ & $6.8(17)$ \\
\hline 4 & $6.6(10)$ & $3.6(11)$ & $7.6(11)$ \\
\hline 5 & $5.2(15)$ & $8.8(12)$ & $1.5(17)$ \\
\hline 6 & $2.6(16)$ & $4.4(13)$ & $7.3(17)$ \\
\hline 7 & $5.2(15)$ & $1.4(13)$ & $6.0(16)$ \\
\hline 8 & $2.6(16)$ & $7.0(13)$ & $3.0(17)$ \\
\hline 9 & $1.3(10)$ & $1.4(11)$ & $1.1(11)$ \\
\hline 10 & $5.0(15)$ & $1.2(13)$ & $6.4(16)$ \\
\hline 11 & $2.5(16)$ & $6.1(13)$ & $3.2(17)$ \\
\hline 12 & $8.9(10)$ & $3.7(11)$ & $7.6(11)$ \\
\hline 13 & $5.2(15)$ & $5.1(12)$ & $1.4(17)$ \\
\hline 14 & $2.6(16)$ & $2.5(13)$ & $6.8(17)$ \\
\hline 15 & $5.2(15)$ & $8.9(12)$ & $1.5(17)$ \\
\hline 16 & $2.6(16)$ & $4.4(13)$ & $7.3(17)$ \\
\hline 17 & 7.1(08) & $4.4(10)$ & $2.0(10)$ \\
\hline 18 & $5.2(15)$ & $5.0(12)$ & $1.4(17)$ \\
\hline 19 & $2.6(16)$ & $2.5(13)$ & $6.8(17)$ \\
\hline 20 & $6.4(09)$ & $1.2(11)$ & $1.1(11)$ \\
\hline 21 & $5.2(15)$ & $8.8(12)$ & $1.5(17)$ \\
\hline 22 & $2.6(16)$ & $4.4(13)$ & $7.3(17)$ \\
\hline 23 & $5.2(15)$ & $1.4(13)$ & $6.0(16)$ \\
\hline 24 & $2.6(16)$ & $7.0(13)$ & $3.0(17)$ \\
\hline 25 & $1.5(09)$ & $4.6(10)$ & $2.0(10)$ \\
\hline 26 & $5.0(15)$ & $1.2(13)$ & $6.4(16)$ \\
\hline 27 & $2.5(16)$ & $6.1(13)$ & $3.2(17)$ \\
\hline 28 & $3.2(06)$ & $8.2(08)$ & $1.1(08)$ \\
\hline 29 & $5.2(15)$ & $4.9(12)$ & $1.3(17)$ \\
\hline 30 & $2.6(16)$ & $2.5(13)$ & $6.8(17)$ \\
\hline 31 & $2.2(11)$ & $2.6(11)$ & $1.4(11)$ \\
\hline 32 & $6.8(12)$ & $5.1(12)$ & $4.2(12)$ \\
\hline 33 & $3.4(13)$ & $2.5(13)$ & $2.1(13)$ \\
\hline 34 & $7.8(11)$ & $5.8(11)$ & $2.5(11)$ \\
\hline 35 & $8.7(12)$ & $6.3(12)$ & $2.9(12)$ \\
\hline 36 & $4.4(13)$ & $3.2(13)$ & $1.4(13)$ \\
\hline 37 & $7.6(11)$ & $6.4(11)$ & $2.0(11)$ \\
\hline 38 & $7.5(12)$ & $6.4(12)$ & $1.9(12)$ \\
\hline 39 & $3.7(13)$ & $3.2(13)$ & $9.7(12)$ \\
\hline 40 & $4.0(10)$ & $4.6(11)$ & $6.2(11)$ \\
\hline 41 & $5.2(15)$ & $2.6(13)$ & $1.6(17)$ \\
\hline 42 & $2.6(16)$ & $1.3(14)$ & 7.9(17) \\
\hline 43 & $5.0(09)$ & $1.6(11)$ & $9.6(10)$ \\
\hline 44 & $5.2(15)$ & $3.2(13)$ & $1.4(17)$ \\
\hline 45 & $2.6(16)$ & $1.6(14)$ & $6.8(17)$ \\
\hline 46 & $5.2(15)$ & $1.4(13)$ & $8.9(16)$ \\
\hline 47 & $2.6(16)$ & 7.7(13) & $4.5(17)$ \\
\hline 48 & $5.2(15)$ & $2.5(13)$ & $1.6(17)$ \\
\hline 49 & $2.6(16)$ & $1.3(14)$ & $7.9(17)$ \\
\hline 50 & $5.2(15)$ & $1.4(13)$ & $8.9(16)$ \\
\hline 51 & $2.6(16)$ & 7.7(13) & $4.5(17)$ \\
\hline 52 & $5.4(10)$ & $4.8(11)$ & $6.2(11)$ \\
\hline 53 & $5.2(15)$ & $3.2(13)$ & $1.4(17)$ \\
\hline 54 & $2.6(16)$ & $1.6(14)$ & $6.8(17)$ \\
\hline 55 & $4.4(09)$ & $1.6(11)$ & $9.7(10)$ \\
\hline 56 & $5.2(15)$ & $2.6(13)$ & $1.6(17)$ \\
\hline 57 & $2.6(16)$ & $1.3(14)$ & 7.9(17) \\
\hline 58 & $1.1(10)$ & $1.2(11)$ & $1.2(11)$ \\
\hline 59 & $5.2(15)$ & $5.0(12)$ & $1.2(17)$ \\
\hline 60 & $2.6(16)$ & $2.5(13)$ & $6.2(17)$ \\
\hline 61 & $5.2(15)$ & $9.5(12)$ & $1.1(17)$ \\
\hline 62 & $2.6(16)$ & $4.7(13)$ & $5.7(17)$ \\
\hline 63 & $5.2(15)$ & $1.9(13)$ & $3.1(16)$ \\
\hline 64 & $2.6(16)$ & $9.6(13)$ & $1.5(17)$ \\
\hline 65 & $5.5(17)$ & $6.4(18)$ & $9.9(16)$ \\
\hline 66 & $6.1(17)$ & $6.2(17)$ & $1.0(17)$ \\
\hline 67 & $8.5(16)$ & $6.6(17)$ & $6.7(16)$ \\
\hline
\end{tabular}


A\&A 607, A118 (2017)

Table A.5. Column densities at $10^{6} \mathrm{yr}$ for other selected species. $a(b)$ stands for $a \times 10^{b}$.

\begin{tabular}{|c|c|c|c|c|c|}
\hline$M$ & $N\left(\mathrm{CH}_{3} \mathrm{OH}\right)$ & $N(\mathrm{SiO})$ & $\begin{array}{c}N(\mathrm{HNC}) \\
\mathrm{cm}^{-2}\end{array}$ & $N(\mathrm{SO})$ & $N(\mathrm{HNCO})$ \\
\hline 2 & $2.8(14)$ & $4.7(15)$ & $1.7(15)$ & $1.3(17)$ & $8.0(11)$ \\
\hline 3 & $6.4(14)$ & $2.2(16)$ & $5.7(15)$ & $4.9(17)$ & $4.2(13)$ \\
\hline 5 & $1.5(14)$ & $4.0(15)$ & $2.2(15)$ & $1.5(15)$ & $1.3(15)$ \\
\hline 6 & 2.9(15) & $1.7(16)$ & $3.0(16)$ & $2.1(15)$ & $6.2(15)$ \\
\hline 7 & $3.3(14)$ & $2.6(15)$ & $5.9(15)$ & $2.4(14)$ & 5.1(14) \\
\hline 8 & $1.2(15)$ & $1.4(16)$ & $1.9(15)$ & $2.2(15)$ & $6.3(15)$ \\
\hline 18 & $2.6(14)$ & $4.7(15)$ & $1.7(15)$ & $1.3(17)$ & $7.9(11)$ \\
\hline 19 & $6.4(14)$ & $2.2(16)$ & $5.7(15)$ & $4.9(17)$ & $4.2(13)$ \\
\hline 21 & $1.3(14)$ & $4.0(15)$ & $2.2(15)$ & $1.6(15)$ & $1.3(15)$ \\
\hline 22 & $2.9(15)$ & $1.7(16)$ & $3.0(16)$ & $2.1(15)$ & $6.2(15)$ \\
\hline 23 & $3.1(14)$ & $2.6(15)$ & $5.8(15)$ & $2.4(14)$ & $5.5(14)$ \\
\hline 24 & $1.2(15)$ & $1.4(16)$ & $1.9(15)$ & $2.2(15)$ & $6.3(15)$ \\
\hline 29 & $2.3(12)$ & $4.9(15)$ & $1.7(15)$ & $1.3(17)$ & $6.5(11)$ \\
\hline 30 & $6.4(14)$ & $2.2(16)$ & $5.7(15)$ & $4.9(17)$ & $4.2(13)$ \\
\hline 41 & $1.3(14)$ & $4.0(15)$ & $5.6(15)$ & $6.8(15)$ & $1.0(15)$ \\
\hline 42 & $3.7(15)$ & $1.7(16)$ & $9.1(16)$ & $1.6(16)$ & $1.7(16)$ \\
\hline 44 & $3.3(14)$ & $4.7(15)$ & $2.9(15)$ & $1.3(17)$ & $1.8(12)$ \\
\hline 45 & $3.6(14)$ & $2.1(16)$ & $9.6(15)$ & $5.0(17)$ & 7.1(13) \\
\hline 46 & $5.0(14)$ & $2.3(15)$ & $1.3(16)$ & $1.6(15)$ & 6.1(14) \\
\hline 47 & $6.4(14)$ & $1.4(16)$ & $4.5(16)$ & $2.1(16)$ & $7.5(15)$ \\
\hline 50 & $4.8(14)$ & $2.3(15)$ & $1.2(16)$ & $1.8(15)$ & $6.5(14)$ \\
\hline 51 & $6.4(14)$ & $1.4(16)$ & $4.5(16)$ & $2.1(16)$ & $7.5(15)$ \\
\hline 56 & $1.2(14)$ & $4.0(15)$ & $5.5(15)$ & 7.1(15) & $9.5(14)$ \\
\hline 57 & $3.7(15)$ & $1.7(16)$ & $9.1(16)$ & $1.6(16)$ & $1.7(16)$ \\
\hline 59 & $2.1(14)$ & $4.7(15)$ & $1.5(15)$ & $1.2(17)$ & $5.7(11)$ \\
\hline 60 & $1.3(15)$ & $2.2(16)$ & $5.3(15)$ & $4.8(17)$ & $3.5(13)$ \\
\hline 61 & $1.4(14)$ & $4.0(15)$ & $2.0(15)$ & $3.6(14)$ & $1.7(15)$ \\
\hline 62 & $2.0(15)$ & $1.7(16)$ & $2.6(16)$ & $6.7(14)$ & $8.6(15)$ \\
\hline 63 & $2.7(14)$ & $2.0(15)$ & $2.6(15)$ & 7.7(13) & $9.9(14)$ \\
\hline 64 & $8.4(14)$ & $1.4(16)$ & $8.8(14)$ & $4.2(14)$ & $9.3(15)$ \\
\hline
\end{tabular}

